\title{
"Simplicity is complexity resolved": the case of postoperative radiation therapy after breast conservation
}

\author{
Pierfrancesco Franco $^{1}$, Gary M. Freedman ${ }^{2}$, Umberto Ricardi $^{1}$, Philip Poortmans ${ }^{3}$ \\ ${ }^{1}$ Department of Oncology-Radiation Oncology, University of Turin, Turin, Italy; ${ }^{2}$ Department of Radiation Oncology, Perelman School of Medicine \\ of the University of Pennsylvania, Philadelphia, Pennsylvania, USA; ${ }^{3}$ Department of Radiation Oncology, Radboud University Medical Center, \\ Nijmegen, The Netherlands \\ Correspondence to: Pierfrancesco Franco, MD. Department of Oncology-Radiation Oncology, University of Turin School of Medicine, Via Genova 3, \\ 10126, Turin, Italy. Email: pierfrancesco.franco@unito.it. \\ Comment on: Killander F, Karlsson P, Anderson H, et al. No breast cancer subgroup can be spared postoperative radiotherapy after breast-conserving \\ surgery. Fifteen-year results from the Swedish Breast Cancer Group randomised trial, SweBCG 91 RT. Eur J Cancer 2016;67:57-65.
}

Submitted Nov 03, 2016. Accepted for publication Nov 16, 2016.

doi: $10.21037 /$ tcr.2016.12.75

View this article at: http://dx.doi.org/10.21037/tcr.2016.12.75

"Simplicity is complexity resolved"-Constantin Brancusi

Postoperative whole breast radiation therapy (WBRT) after breast conserving surgery (BCS) is considered the standard of care for early-stage breast cancer (EBC) patients. In recent population-based studies long-term overall survival (OS) might even be better than that following mastectomy without radiotherapy (RT) $(1,2)$. Overall, WBRT halves the 10 -year rate of any breast cancer recurrence and reduces by about one sixth the 15 -year breast cancer-related mortality (3). Nevertheless, the absolute reduction in the rate of relapse and cancer mortality are to some extent proportionally affected by patient- and tumor-related characteristics including age, grade, nodal involvement and estrogen receptor status, calling for the need of tailoring clinical indications for WBRT (3).

Killander et al. recently reported the long-term results of the SweBCG 91 RT randomized phase III trial, investigating the option to omit WBRT for selected EBC (4). Clinical T1-T2N0M0 patients were randomly assigned to receive or not WBRT (48-54 Gy in 24-27 fractions) after BCS. Adjuvant systemic therapy was prescribed for stage II patients according to regional treatment guidelines. Among the 1,187 randomized patients, only 84 received tamoxifen (47 vs. 37 in the control and RT arms, respectively) and 22 CMF chemotherapy (13 vs. 9) (4). After a median followup of 15.6 years, patients without WBRT had a significantly higher ipsilateral breast tumor recurrence risk (IBTR) (23.9\%), compared to irradiated patients $(11.5 \%)$ and a lower recurrence-free survival (51.7\% vs. $60.4 \%)$. OS did not significantly differ (68.4\% vs. $71.1 \%)$ (4).

The role of WBRT after BCS has been extensively documented in several historical randomized studies, carried out both in Europe and in the USA, showing a significant increase in IBTR rates for patients not receiving RT (5-9). Local relapses at 5 years were observed to rise from $2-20 \%$ with WBRT to $27-42 \%$ without. However, none of these trials, including SweBCG $91 \mathrm{RT}$, were able to demonstrate an OS benefit by the addition of WBRT. Moreover, the observation that certain subsets of patients may have a lower risk of IBTR according to specific tumor- and patient-related characteristics, led to initiatives to selectively decrease the overall therapeutic burden in tailored subgroups of EBC (10). As for example in the Milan III trial, the IBTR rates in the no WBRT group were $7.4 \%$ at 10 years below 45 years of age, $3.1 \%$ in the age range $46-55$ years, $1.7 \%$ for $56-65$ years and equal to the WBRT arm over 65 years, age was often used as the most important discriminating factor (11).

Ideally, individualized cancer therapy should allow for treatment option selection according to clinical as well as biological characteristics, favoring the minimally required therapeutic package to achieve adequate tumor control with acceptable toxicity and optimal long-term quality of life (12). This is particularly topical in "lowrisk" breast cancer patients. Therefore, several studies investigated the potentials of de-intensification of treatment in this subgroup. In the CALGB 9343 trial, women aged 
$\geq 70$ years with estrogen-receptor positive and clinical stage I (cT1N0M0) disease were randomized to receive WBRT + tamoxifen or exclusive tamoxifen as adjuvant treatment after BCS. Multiple endpoints were investigated (time to local and regional recurrence, rate of salvage mastectomies, time to distant failure and breast cancer-specific and OS). At 5 years, the IBTR rate was higher for patients having WBRT omitted (4\% vs. 1\%), while all other endpoints were not significantly different. The arm submitted to tamoxifen alone had better cosmetic outcomes and lower adverse effects (13). Similar findings were observed in the PRIME II study, where low-risk EBC patients aged 65 years or older [positive hormone receptors; axillary node negative; clinical T1-T2 disease up to $3 \mathrm{~cm}$, grade 3 or lymphovascular invasion (LVI) but not both, and clear margins after BCS] and receiving adjuvant endocrine therapy were randomized to conventionally fractionated or hypofractionated WBRT $v s$. no radiation (14). The rates of IBTR were $1.3 \%$ for WBRT and $4.1 \%$ for the no radiation arm at 5 years, with a global hazard ratio (HR) of 5.19. Regional, distant and contralateral breast relapses and breast cancer-related mortality were not different. An Italian study, with similar design and a 9-year median observation time, found similar rates of IBTR (3.4 for WBRT vs. 4.4 for no radiation) and OS $(81.4 \%$ vs. $83.7 \%)$ in unifocal breast cancer $<25 \mathrm{~mm}$, with $0-3$ positive axillary nodes and no extensive intraductal component or LVI (15).

Notwithstanding these studies, the identification of the most appropriate EBC category to be offered deintensification with WBRT omission remains a challenge. This is confirmed by the data reported in the SweBCG 91 RT trial, where the 15 -year cumulative incidence of IBTR in patients without WBRT ranged from $16.7 \%$ to $28 \%$ among subgroups within different categories based on age, tumor size, hormonal receptor status and method of diagnosis (4). More interestingly, in low-risk patients ( $>64$ years, $<21 \mathrm{~mm}$ sized tumor, positive oestrogen and progesterone receptor) the cumulative reduction in IBTR rates following WBRT was even higher than in the whole population (IBRT: $25.9 \%$ for no WBRT arm vs. $5.3 \%$ for the radiation arm). The explanation why no influence of the omission of WBRT on OS was detected might be that follow-up is still too short, the low intensity of adjuvant systemic therapy given initially and/or the effectiveness of salvage therapy after BCS alone. Moreover, elderly low-risk EBC form a particular population with a high burden of competing causes of death. However, in general, preventing a local relapse has clinical meaningfulness (3) and there is a need for a careful clinical decision-making process before omitting WBRT, even in low-risk EBC and even without demonstration of a clear impact of WBRT on OS in this setting.

The importance of endocrine therapy in patients having WBRT omitted was shown in the German Breast Study Group (GBSG) trial, where the crude risk of local recurrence at 10 years was $7-10 \%$ in patients treated either with BCS and WBRT, BCS and tamoxifen, or BCS and both, but was $34 \%$ in those treated with BCS alone (16). However, as treatment safety and quality of life are also crucial endpoints, we should also question the need for adjuvant systemic therapy in low-risk EBC. Endocrine therapy may be associated with an increased risk for osteoporosis with skeletal related events, cardiovascular disease, sexual dysfunction and even neurocognitive effects (10). Moreover the impact of hormonal therapy in terms of OS in post-menopausal patients has yet to be confirmed with even compliance to treatment being challenging, as only $35-60 \%$ of women accomplish a full 5 -year adjuvant program (10). Overall, the side-effects of adjuvant systemic therapy are expected to outweigh those of WBRT, especially as new developments in the field of radiation oncology substantially decreased the burden of radiation therapy (10). Hypofractionated schedules of WBRT and accelerated partial breast irradiation provide new options to decrease overall treatment time, cost, inconvenience, and toxicity of postoperative radiation (17-19).

The results of the SweBCG $91 \mathrm{RT}$ trial and others confirm that clinical and pathologic selection of seemingly low-risk features like age for omission of radiation have reached their limit of usefulness. A selection based on biology of the tumor may be more fruitful in the future. Liu et al. conducted a retrospective subgroup analysis on 501 of 769 available blocks from the larger Canadian prospective trial that had shown a significant benefit to WBRT after BCS and tamoxifen (20). They conducted intrinsic subtyping and showed that luminal subtypes seemed to derive less benefit from WBRT (luminal A HR: 0.40; luminal B HR: 0.51) than high-risk subtypes of HER-2 or triple negative (HR: 0.13), however without reaching statistical significance. Additional studies in North America and Europe that will study the use of subtyping for EBC in clinically low-risk women by receptor expression, genomic expression or signature assays to study omission of radiation include the LUMINA, IDEA, PRECISION and PRIMITIME trials (21).

Conclusion: the selection of the most appropriate 
treatment for low-risk breast cancer patients should take into account not only risk for local relapse or impact on survival, but also the treatment-related toxicity profile, patient quality of life, psycho-social issues and costeffectiveness. Biological and molecular patterns should be investigated to assist in the clinical decision-making process. Composite endpoints and validated evaluation tools are mandatory for comparative evaluation of treatments and appropriate allocation of future patients. The quest to identify which EBC patients can forego WBRT after BCS remains to be accomplished. Simple questions may lead to complicated answers but using a holistic approach on therapeutic modalities and clinical endpoints we may achieve simplicity through a resolution of complexity, with a consequent benefit for breast cancer patients.

\section{Acknowledgments}

Funding: None.

\section{Footnote}

Provenance and Peer Review: This article was commissioned and reviewed by the Section Editor San-Gang Wu (Department of Radiation Oncology, Xiamen Cancer Center, the First Affiliated Hospital of Xiamen University, Xiamen, China).

Conflicts of Interest: All authors have completed the ICMJE uniform disclosure form (available at http://dx.doi. org/10.21037/tcr.2016.12.75). The authors have no conflicts of interest to declare.

Ethical Statement: The authors are accountable for all aspects of the work in ensuring that questions related to the accuracy or integrity of any part of the work are appropriately investigated and resolved.

Open Access Statement: This is an Open Access article distributed in accordance with the Creative Commons Attribution-NonCommercial-NoDerivs 4.0 International License (CC BY-NC-ND 4.0), which permits the noncommercial replication and distribution of the article with the strict proviso that no changes or edits are made and the original work is properly cited (including links to both the formal publication through the relevant DOI and the license). See: https://creativecommons.org/licenses/by-nc-nd/4.0/.

\section{References}

1. Poortmans P. Evidence based radiation oncology: breast cancer. Radiother Oncol 2007;84:84-101.

2. van Maaren MC, de Munck L, de Bock GH, et al. 10 year survival after breast-conserving surgery plus radiotherapy compared with mastectomy in early breast cancer in the Netherlands: a population-based study. Lancet Oncol 2016;17:1158-70.

3. Early Breast Cancer Trialists' Collaborative Group (EBCTCG), Darby S, McGale P, et al. Effect of radiotherapy after breast-conserving surgery on 10year recurrence and 15-year breast cancer death: metaanalysis of individual patient data for 10,801 women in 17 randomised trials. Lancet 2011;378:1707-16.

4. Killander F, Karlsson P, Anderson H, et al. No breast cancer subgroup can be spared postoperative radiotherapy after breast-conserving surgery. Fifteen-year results from the Swedish Breast Cancer Group randomised trial, SweBCG 91 RT. Eur J Cancer 2016;67:57-65.

5. Veronesi U, Luini A, Del Vecchio M, et al. Radiotherapy after breast-preserving surgery in women with localized cancer of the breast. N Engl J Med 1993;328:1587-91.

6. Forrest AP, Stewart HJ, Everington D, et al. Randomised controlled trial of conservation therapy for breast cancer: 6-year analysis of the Scottish trial. Scottish Cancer Trials Breast Group. Lancet 1996;348:708-13.

7. Liljegren G, Holmberg L, Bergh J, et al. 10-Year results after sector resection with or without postoperative radiotherapy for stage I breast cancer: a randomized trial. J Clin Oncol 1999;17:2326-33.

8. Fisher B, Bryant J, Dignam JJ, et al. Tamoxifen, radiation therapy, or both for prevention of ipsilateral breast tumor recurrence after lumpectomy in women with invasive breast cancers of one centimeter or less. J Clin Oncol 2002;20:4141-9.

9. Clark RM, Whelan T, Levine M, et al. Randomized clinical trial of breast irradiation following lumpectomy and axillary dissection for node-negative breast cancer: an update. Ontario Clinical Oncology Group. J Natl Cancer Inst 1996;88:1659-64.

10. Poortmans PM, Arenas M, Livi L. Over-irradiation. Breast 2016. [Epub ahead of print].

11. Veronesi U, Marubini E, Mariani L, et al. Radiotherapy after breast-conserving surgery in small breast carcinoma: long-term results of a randomized trial. Ann Oncol 2001;12:997-1003.

12. Veronesi U, Stafyla V, Luini A, et al. Breast cancer: from 
"maximum tolerable" to "minimum effective" treatment. Front Oncol 2012;2:125.

13. Hughes KS, Schnaper LA, Berry D, et al. Lumpectomy plus tamoxifen with or without irradiation in women 70 years of age or older with early breast cancer. N Engl J Med 2004;351:971-7.

14. Kunkler IH, Williams LJ, Jack WJ, et al. Breast-conserving surgery with or without irradiation in women aged 65 years or older with early breast cancer (PRIME II): a randomised controlled trial. Lancet Oncol 2015;16:266-73.

15. Tinterri C, Gatzemeier W, Costa A, et al. Breastconservative surgery with and without radiotherapy in patients aged 55-75 years with early-stage breast cancer: a prospective, randomized, multicenter trial analysis after 108 months of median follow-up. Ann Surg Oncol 2014;21:408-15.

16. Winzer KJ, Sauer R, Sauerbrei W, et al. Radiation therapy after breast-conserving surgery; first results of a randomised clinical trial in patients with low risk of recurrence. Eur J Cancer 2004;40:998-1005.

17. Freedman GM, White JR, Arthur DW, et al. Accelerated

Cite this article as: Franco P, Freedman GM, Ricardi U, Poortmans P. "Simplicity is complexity resolved": the case of postoperative radiation therapy after breast conservation. Transl Cancer Res 2016;5(Suppl 7):S1336-S1339. doi: 10.21037/ tcr.2016.12.75 fractionation with a concurrent boost for early stage breast cancer. Radiother Oncol 2013;106:15-20.

18. Shaitelman SF, Khan AJ, Woodward WA, et al. Shortened radiation therapy schedules for early-stage breast cancer: a review of hypofractionated whole-breast irradiation and accelerated partial breast irradiation. Breast J 2014;20:131-46.

19. Franco P, Zeverino M, Migliaccio F, et al. Intensitymodulated and hypofractionated simultaneous integrated boost adjuvant breast radiation employing statics ports of tomotherapy (TomoDirect): a prospective phase II trial. J Cancer Res Clin Oncol 2014;140:167-77.

20. Liu FF, Shi W1, Done SJ, et al. Identification of a Low-Risk Luminal A Breast Cancer Cohort That May Not Benefit From Breast Radiotherapy. J Clin Oncol 2015;33:2035-40.

21. Kirwan CC, Coles CE, Bliss J, et al. It's PRIMETIME. Postoperative Avoidance of Radiotherapy: Biomarker Selection of Women at Very Low Risk of Local Recurrence. Clin Oncol (R Coll Radiol) 2016;28:594-6. 